\title{
Mobile Bearing Plate Dislocation in Total Knee Arthroplasty Due to Muscle Spasm: A Case Report
}

\author{
Jean-Damien Nicodeme ${ }^{1}$, Claus Löcherbach ${ }^{1}$, Alexandre Terrier ${ }^{2}$, Aurélien Gallice ${ }^{2}$, Brigitte M. Jolles ${ }^{1}$ \\ ${ }^{1}$ Department of Orthopaedic Surgery and Traumatology, Centre Hospitalier Universitaire Vaudois and University of Lausanne, \\ Lausanne, Switzerland; ${ }^{2}$ Laboratory of Biomechanical Orthopedics, Interinstitutional Center of Translational Biomechanics, Ecole \\ Polytechnique Fédérale de Lausanne, Lausanne, Switzerland. \\ Email: Brigitte.jolles-haeberli@chuv.ch
}

Received March $22^{\text {nd }}, 2013$; revised May $1^{\text {st }}, 2013$; accepted May $15^{\text {th }}, 2013$

Copyright (c) 2013 Jean-Damien Nicodeme et al. This is an open access article distributed under the Creative Commons Attribution License, which permits unrestricted use, distribution, and reproduction in any medium, provided the original work is properly cited.

\begin{abstract}
An unusual case of early dislocation of a mobile bearing posterior stabilized total knee arthroplasty in a 48-year-old Caucasian woman is described. Dislocation occurred one day postoperatively, attributed to a gap mismatch. Revision surgery reduced posterior dislocation, increased bearing plate thickness and rebalanced ligaments. A second dislocation occurred after revision surgery. The patient's history was retaken and a hamstring spasm disease identified. A new revision utilized a more constrained design, without perioperative local nerve block. Two years following surgery, no further dislocation had occurred. A numerical musculoskeletal model of the case and implant configuration identified no trend to mobile bearing dislocation when regular muscle forces were applied. Muscle spasm is a risk factor for mobile bearing total knee arthroplasty dislocation, especially with femoral nerve block.
\end{abstract}

Keywords: Dislocation; Femoral Nerve Block; Mobile Bearing Prosthesis; Muscle Spasm; Total Knee Arthroplasty

\section{Introduction}

Dislocation following total knee arthroplasty (TKA) is a well known but rare event. The prevalence depends on the type of prosthesis, with a higher rate reported for mobile bearing TKA. Early reports of dislocation rates in mobile bearing designs were high, approaching 10\% [1,2], but modified surgical techniques and improved designs have led to reduced dislocation rates [3]. In the last 10 years, dislocation rates reported for mobile bearing implants varied from $0.4 \%$ to $6.4 \%$ [4-11]. For the fixed bearing design, dislocation rates range from $0 \%$ [5,9,12] to $0.5 \%$ [13]. This complication typically occurs in the early postoperative period, from days to within 2 years after surgery $[1,4,6,9]$.

Stiehl [14] considered dislocation following TKA a consequence of limb malalignment (valgus or varus) or flexion imbalance. In addition to limb deformity [6,7,11], flexion imbalance $[4,8]$ and prosthesis design, other factors that have been implicated in dislocation include tibial component malposition [10,15], patellar dysfunction [16], extensive posterolateral release or increased femorotibial laxity [4,6,10,14], and myelopathy [17].

We report the case of a posterior dislocation in a mo- bile bearing TKA where hamstring spasms and local perioperative anesthesia are implicated.

\section{Case Report}

A 48-year-old Caucasian woman, $158 \mathrm{~cm}$ tall and weighing $99 \mathrm{~kg}$, with body mass index of 39, presented with ongoing left knee pain that occurred after walking more than 2 blocks and when climbing stairs, with no history of trauma. She complained of severe limitation in daily life.

Patient history revealed spondylodesis of L4-L5 and L5-S1 in 1976, deep venous thrombosis of the left leg in 2005, and a family history of early osteoarthritis.

Clinical examination revealed the patient did not limp and had full knee extension and $100^{\circ}$ of flexion limited by abundant soft tissue. Limb alignment showed a partially reducible varus deformity. No joint effusion was observed. Ligament testing revealed medial compartment pseudolaxity. Patellar tracking was normal, as were neurological and vascular examinations.

Radiological examination consisting of antero-posterior and lateral views at $30^{\circ}$ of flexion (Figure 1), Rosenberg view, long standing radiography and patellar 
view confirmed three compartment, Ahlbäck stage III osteoarthritis with $5^{\circ}$ varus deformity (Figure 2(a)). Medical treatment with anti-inflammatory medications and physiotherapy was ineffective. We therefore recommended a total knee replacement.

An ultra-congruent postero-stabilized mobile bearing implant (F.I.R.S.T., Symbios Orthopédie, CH) was used. Surgery proceeded (using a medial parapatellar approach) without complications and intraoperative knee stability was good. Flexion extension gap balancing was perfect upon closing. Postoperative radiological assessment showed correct implant position. Standard rehabilitation was initiated. During the second re-education session the patient complained of severe pain and a feeling of a locking knee. Radiography showed a posterior dislocation of the tibia with $90^{\circ}$ rotation of the tibial bearing plate (Figure 3).

The patient underwent open reduction and revision surgery on the third postoperative day. Due to a complete tear of the popliteal ligament and partial tear of the lateral collateral ligament along the lateral portion, a medial release was performed to rebalance the knee, and the width of the polyethylene insert was increased from 11 $\mathrm{mm}$ to $17 \mathrm{~mm}$. Intraoperative assessment confirmed a posterior dislocation of the tibia with extreme external rotation of the mobile bearing plate and a tear of the popliteal tendon. Testing at the end of surgery showed proper ligament balance and good stability in flexion and extension. The knee was placed in a Velcro knee splint after surgery. Standard postoperative analgesia consisted of a femoral nerve block and intravenous opioids.

Postoperative radiographs taken approximately 2 hours after surgery showed a recurrent posterior dislocation of the tibia. The patient's history was retaken, and a hamstring spasm disease was identified. A new revision was performed using a more constrained design of the same prosthesis model, with no local perioperative nerve block. At two years follow up, no other dislocation had occurred.

\section{Numerical Study}

The case study was simulated with a numerical musculoskeletal model of the knee joint developed in Abaqus (9) that included the femur, tibia, patella, quadriceps (divided into the vastus medialis, vastus lateralis, vastus medialis and rectus femoris), hamstring (divided into the biceps femoris, semitendinosus and semimembranosus), and cruciate and collateral ligaments. The total knee prosthesis was virtually inserted into the knee model, with $11 \mathrm{~mm}$ and $17 \mathrm{~mm}$ polyethylene inserts tested sequentially. Two knee flexion angles (30 and 50 degrees) were investigated, where the femur was fixed while the tibia was allowed to translate in infero-superior and antero-posterior directions, and tibio-femoral and pa-

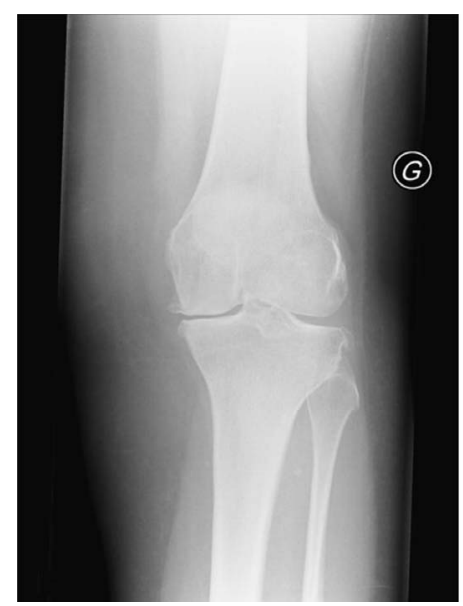

(a)

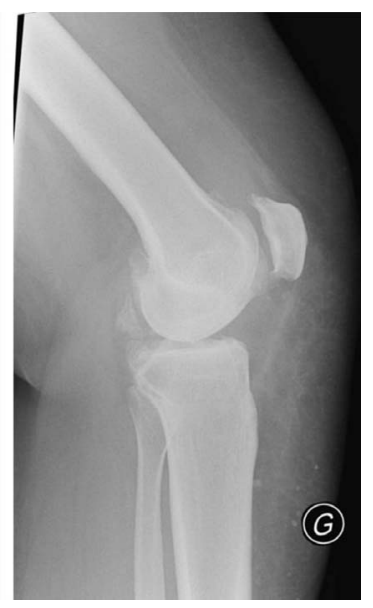

(b)
Figure 1. Preoperative radiographs of left knee, with three compartment, Ahlbäck stage III osteoarthritis with $5^{\circ}$ varus deformity: (a) Anteroposterior view; (b) Lateral view at $30^{\circ}$ of flexion.

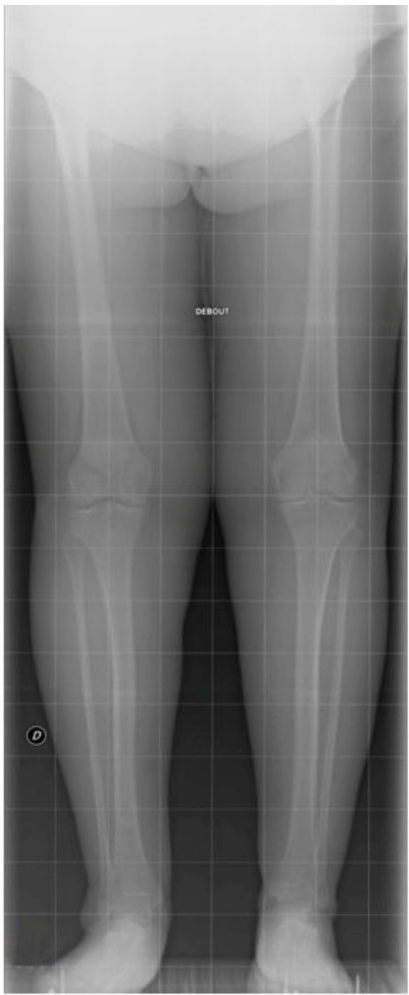

(a)

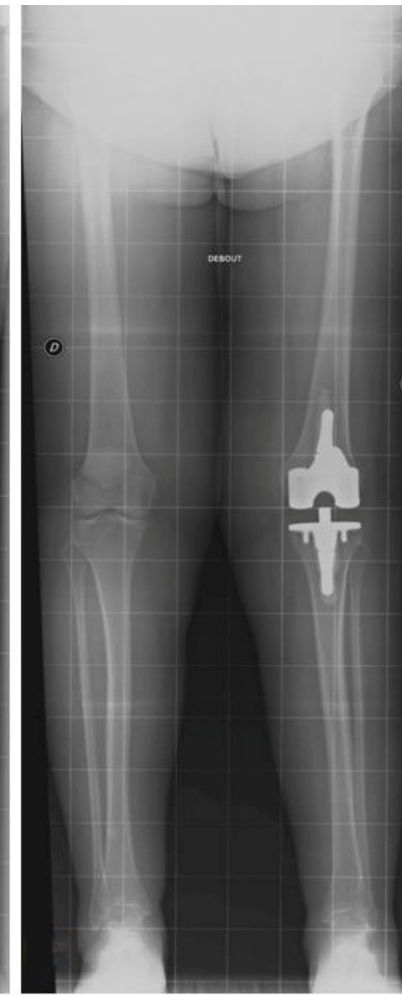

(b)
Figure 2. Standing radiographs, antero-posterior views (a) preoperative, left leg in $5^{\circ}$ varus; (b) 6 weeks following second revision, left leg in $2^{\circ}$ valgus.

tello-femoral contacts were considered. The patella was constrained only by its contact with the femoral component and by muscular forces. The muscular spasm was simulated by an increasing force on the hamstring muscles, referred to as the subluxation force, balanced by a 


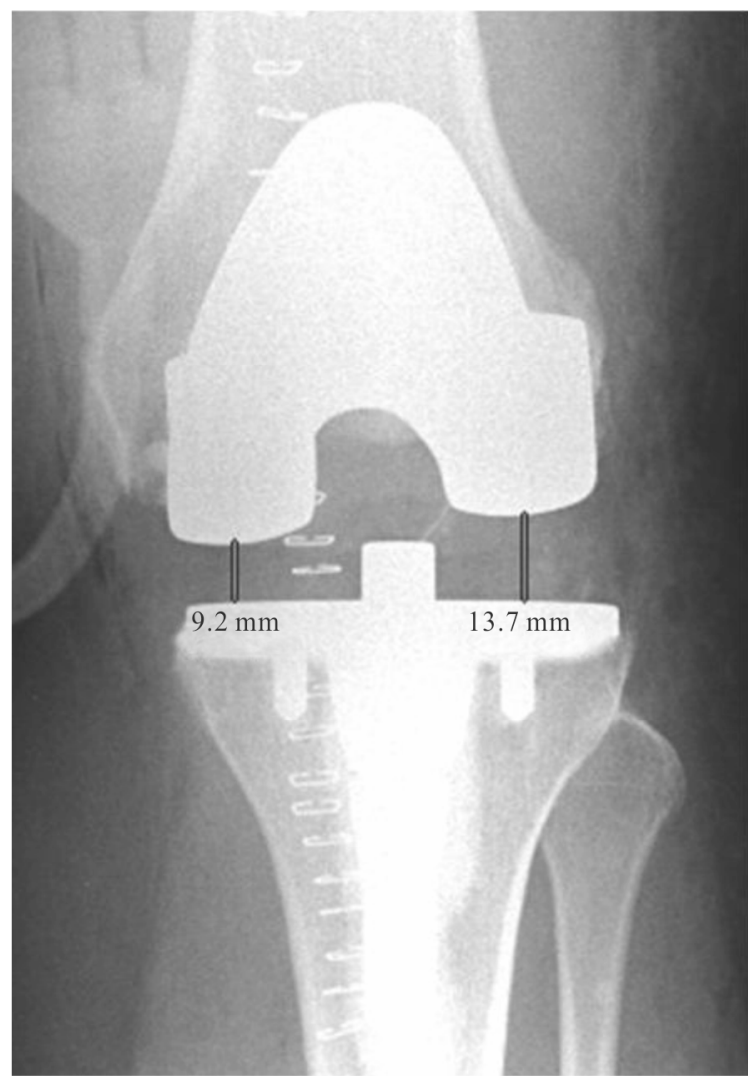

(a)

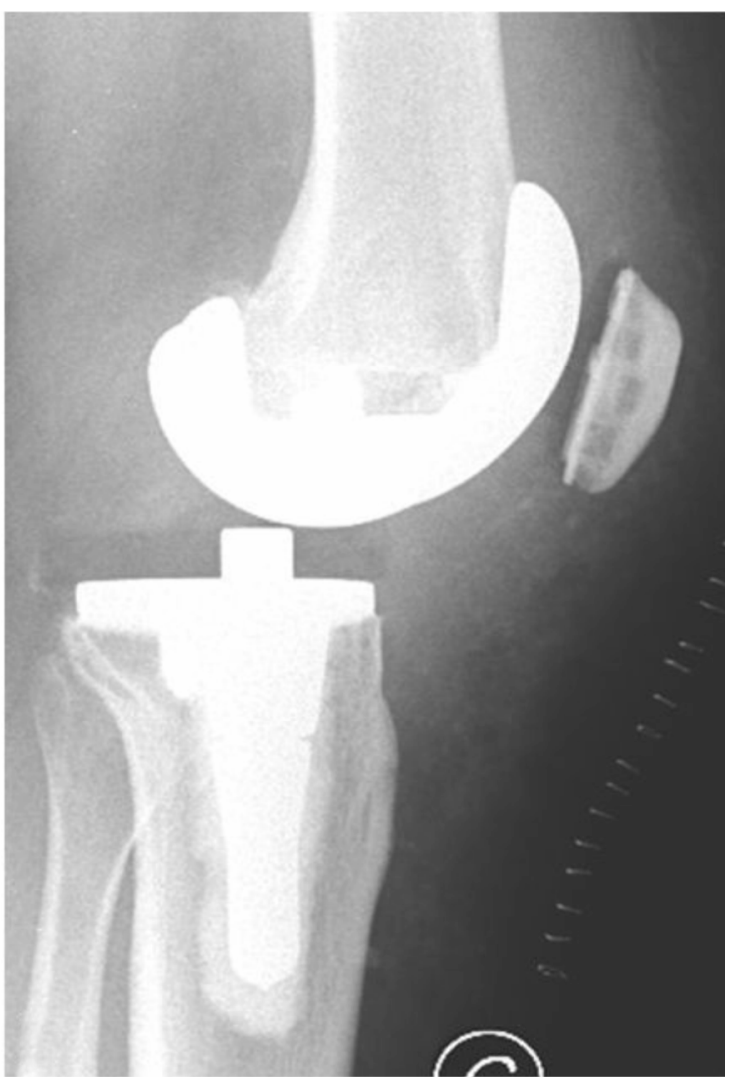

(b)

Figure 2. Radiographs of left knee following total knee arthroplasty, using an ultra-congruent postero-stabilized mobile bearing implant, at 48 hours postoperative. Note posterior dislocation of the tibia with rotation of the tibial bearing plate: (a) Antero-posterior view; (b) Lateral view.

passive reaction of the quadriceps muscles. When posterior dislocation occurred, the antero-posterior component of the tibio-femoral contact reaction force reached a peak value before a rapid drop. This model predicted that dislocation was not possible at 30 degrees of flexion, with either polyethylene insert (11 $\mathrm{mm}$ or $17 \mathrm{~mm})$. At 50 degrees of flexion, dislocation was initiated from a hamstring force of about $650 \mathrm{~N}$, which compares favorably with the $250 \mathrm{~N}$ force required for knee dislocation in vitro [18]. The effect of the polyethylene thickness on dislocation force was less than $2 \%$. At greater angles of flexion, the cam-post mechanism was engaged and dislocation was more difficult.

\section{Discussion}

This case study implicates muscle spasm and local perioperative anaesthesia as novel causes for dislocation following mobile bearing TKA. Mobile bearing designs present certain advantages according to biomechanical trials $[19,20]$. A highly conforming design with a unidirectional rotation or sliding motion that more closely resembles internal rotation of the tibia reduces bone implant interface stress $[19,21]$. The decreased friction forces and consequent reduction in polyethylene wear [19] may increase the life span of TKA.

Alternatively, the increased mobility imparted by mobile bearing implants could compromise implant stability and increase the risk of dislocation. Although a rare complication, some cases of dislocation have been reported. Buechel and Pappas [22] reported a dislocation rate of 3.2\% in 217 low contact stress knee replacements, and Bert [1] reported 4 dislocations in 43 TKAs (9.3\%). More recent studies of mobile bearing implants reported dislocation rates ranging from less than $0.8 \%[4,5,10,11]$, to $2 \%[6,23]$ and a high of $6.4 \%$ [9]. This variation in incidence could be explained by trial heterogeneity with respect to indication for surgery and implant design or constraint choice. For example, Buechel and Pappas [22] included revision arthroplasty, thus increasing the dislocation rate of $2.8 \%$ for primary arthroplasty.

Several risk factors for dislocation following TKA have been reported. The most frequent is a flexion gap mismatch, often due to an asymmetric posterior cut or flexor/extensor ligament imbalance $[4,8,14,16,24]$. Soft tissue balancing is crucial for TKA stability. Preoperative varus or valgus deformity has also been implicated in an 
increased risk of dislocation [6,7,11,14,25]. In these cases, ligament balancing is sometimes difficult, and cuts could lead to an oblique space, increasing dislocation risk. Patient factors associated with mobile-bearing rotating platform dislocations include female sex, obesity, and preoperative valgus deformity [11]. In the case reported here, the female patient was morbidly obese, with a body mass index of 39, and she presented with a varus deformity that was partially reducible. The additional hamstring spasms associated with restless leg disease that was probably exacerbated by a perioperative femoral nerve block is a novel observation that is likely to have contributed to the dislocation. Finally, several studies cite the prosthesis design as an independent risk factor [2628]. Features that influence stability and may reduce the risk of dislocation include a posterior-stabilization system, anterior lip bearing, post cam mechanism, rotation stop pin, third condylar system, bearing diameter, and tibial slope [17,27-29].

The mobile bearing design is probably more demanding surgically [30] with respect to achieving the correct balance between the intrinsic characteristics of the device and the patient's individual pathology.

\section{Conclusion}

Preoperative clinical evaluation should include a meticulous assessment of ligaments and muscle balance. Preoperative planning should take into account not only limb deformity but also implant limitations. This case demonstrates the importance of conducting a meticulous patient history to elucidate factors that can influence prosthesis type, constraint choice and perioperative procedure. Particular attention should be paid to proper balancing perioperatively and, in cases where muscular tone might be pathological, alternative solutions for perioperative analgesia may be found in collaboration with the anesthesiology team. The surgeon should indicate mobile bearing design with caution for these patients.

\section{Acknowledgements}

The authors thank Dagmar Gross for assistance with preparation of this manuscript and Angelina Poloni and P. Widmer for administrative assistance.

\section{REFERENCES}

[1] J. M. Bert, "Dislocation/Subluxation of Meniscal Bearing Elements after New Jersey Low-Contact Stress Total Knee Arthroplasty," Clinical Orthopaedics \& Related Research, Vol. 254, 1990, pp. 211-215. doi:10.1097/00003086-199005000-00030

[2] H. Pandit, C. Jenkins, D. J. Beard, A. J. Price, H. S. Gill, C. A. Dodd and D. W. Murray, "Mobile Bearing Dislocation in Lateral Unicompartmental Knee Replacement,”
Knee, Vol. 17, No. 6, 2010, pp. 392-397. doi:10.1016/j.knee.2009.10.007

[3] J. C. Vogt and C. Saarbach, "LCS Mobile-Bearing Total Knee Replacement. A 10-Year's Follow-Up Study,” Orthopaedics \& Traumatology, Surgery \& Research, Vol. 95, No. 3, 2009, pp. 177-182. doi:10.1016/j.otsr.2009.02.002

[4] N. W. Thompson, D. S. Wilson, G. W. Cran, D. E. Beverland and J. B. Stiehl, "Dislocation of the Rotating Platform after Low Contact Stress Total Knee Arthroplasty," Clinical Orthopaedics \& Related Research, Vol. 425, 2004, pp. 207-211. doi:10.1097/00003086-200408000-00029

[5] Y. H. Kim, H. K. Kook, and J. S. Kim, "Comparison of Fixed-Bearing and Mobile-Bearing Total Knee Arthroplasties," Clinical Orthopaedics \& Related Research, Vol. 392, 2001, pp. 101-115. doi:10.1097/00003086-200111000-00013

[6] C. H. Huang, H. M. Ma, Y. M. Lee and F. Y. Ho, "LongTerm Results of Low Contact Stress Mobile-Bearing Total Knee Replacements," Clinical Orthopaedics \& Related Research, Vol. 416, 2003, pp. 265-270. doi:10.1097/01.blo.0000093890.12372.46

[7] R. B. Sorrells, J. B. Stiehl and P. E. Voorhorst, "Midterm Results of Mobile-Bearing Total Knee Arthroplasty in Patients Younger than 65 Years," Clinical Orthopaedics \& Related Research, Vol. 390, 2001, pp. 182-189. doi:10.1097/00003086-200109000-00021

[8] M. H. Song, B. H. Kim, S. J. Ahn, S. H. Yoo and M. S. Lee, "Early Complications after Minimally Invasive Mobile-Bearing Medial Unicompartmental Knee Arthroplasty,” Journal of Arthroplasty, Vol. 24, No. 8, 2009, pp. 1281-1284. doi:10.1097/00003086-200111000-00013

[9] R. E. Gleeson, R. Evans, C. E. Ackroyd, J. Webb and J. H. Newman, "Fixed or Mobile Bearing Unicompartmental Knee Replacement? A Comparative Cohort Study,” Knee, Vol. 11, No. 5, 2004, pp. 379-384. doi:10.1016/j.knee.2004.06.006

[10] W. S. Cho, Y. S. Youm, S. C. Ahn and D. W. Sohn, "What Have We Learned from LCS Mobile-Bearing Knee System?” Knee Surgery Sports Traumatology Arthroscopy, Vol. 18, No. 10, 2010, pp. 1345-1351. doi:10.1007/s00167-010-1166-5

[11] D. A. Fisher, T. L. Bernasek, R. D. Puri and M. L. Burgess, "Rotating Platform Spinouts with Cruciate-Retaining Mobile-Bearing Knees,” Journal of Arthroplasty, Vol. 26, No. 5, 2011, pp. 877-882. doi:10.1016/j.arth.2011.03.007

[12] J. Insall, W. N. Scott and C. S. Ranawat, "The Total Condylar Knee Prosthesis. A Report of Two Hundred and Twenty Cases," Journal of Bone and Joint Surgery (American), Vol. 61, No. 2, 1979, pp. 173-180.

[13] V. Lombardi Jr., T. H. Mallory, B. K. Vaughn, R. Krugel, T. K. Honkala, M. Sorscher and M. Kolczun, "Dislocation Following Primary Posterior-Stabilized Total Knee Arthroplasty,” Journal of Arthroplasty, Vol. 8, No. 6, 1993, pp. 633-639. doi:10.1016/0883-5403(93)90012-S

[14] J. B. Stiehl, "Mobile Bearings in Total Knee Arthro- 
plasty,” In: W. N. Scott, Ed., Insall \& Scott Surgery of the Knee, 4th Edition, Churchill Livingstone Elsevier, Philadelphia, 2006, pp. 1558-1573.

[15] J. H. Bargren, "Total Knee Dislocation Due to Rotatory Malalignment of Tibial Component: A Case Report," Clinical Orthopaedics \& Related Research, Vol. 147, 1980, pp. 271-274.

[16] P. F. Sharkey, W. J. Hozack, R. E. Booth Jr., R. A. Balderston and R. H. Rothman, "Posterior Dislocation of Total Knee Arthroplasty,” Clinical Orthopaedics \& Related Research, Vol. 278, 1992, pp. 128-133. doi:10.1097/00003086-199205000-00021

[17] M. Hasegawa, A. Sudo, A. Fukuda and A. Uchida, "Dislocation of Posterior-Stabilized Mobile-Bearing Knee Prosthesis. A Case Report,” Knee, Vol. 13, No. 6, 2006, pp. 478-482. doi:10.1016/j.knee.2006.07.002

[18] E. Weale, J. Feikes, D. Prothero, J. J. O’Connor, D. Murray and J. Goodfellow, "In Vitro Evaluation of the Resistance to Dislocation of a Meniscal-Bearing Total Knee Prosthesis between 30 Degrees and 90 Degrees of Knee Flexion,” Journal of Arthroplasty, Vol. 17, No. 4, 2002, pp. 475-483. doi:10.1054/arth.2002.31246

[19] G. W. Blunn, P. S. Walker, A. Joshi and K. Hardinge, "The Dominance of Cyclic Sliding in Producing Wear in Total Knee Replacements," Clinical Orthopaedics \& Related Research, Vol. 273, 1991, pp. 253-260. doi:10.1097/00003086-199112000-00036

[20] J. A. Szivek, P. L. Anderson and J. B. Benjamin, “Average and Peak Contact Stress Distribution Evaluation of Total Knee Arthroplasties," Journal of Arthroplasty, Vol. 11, No. 8, 1996, pp. 952-963. doi:10.1016/S0883-5403(96)80137-9

[21] H. P. Delport, S. A. Banks, J. De Schepper and J. Bellemans, "A Kinematic Comparison of Fixed- and MobileBearing Knee Replacements,” Journal of Bone and Joint Surgery (British), Vol. 88, No. 8, 2006, pp. 1016-1021. doi:10.1302/0301-620X.88B8.17529

[22] F. F. Buechel and M. J. Pappas, "New Jersey Low Contact Stress Knee Replacement System. Ten-Year Evalua- tion of Meniscal Bearings," Orthopedic Clinics of North America, Vol. 20, No. 2, 1989, pp. 147-177.

[23] J. Sanchez-Sotelo, J. M. Ordonez and S. B. Prats, "Results and Complications of the Low Contact Stress Knee Prosthesis,” Journal of Arthroplasty, Vol. 14, No. 7, 1999 , pp. 815-821.

[24] J. B. Stiehl, "World Experience with Low Contact Stress Mobile-Bearing Total Knee Arthroplasty: A Literature Review,” Orthopedics, Vol. 25, No. 2 Suppl, 2002, pp. s213-s217.

[25] B. J. Galinat, J. V. Vernace, R. E. Booth Jr. and R. H. Rothman, "Dislocation of the Posterior Stabilized Total Knee Arthroplasty. A Report of Two Cases,” Journal of Arthroplasty, Vol. 3, No. 4, 1988, pp. 363-367. doi:10.1016/S0883-5403(88)80039-1

[26] J. Chouteau, J. L. Lerat, R. Testa, B. Moyen, M. H. Fessy and S. A. Banks, "Mobile-Bearing Insert Translational and Rotational Kinematics in a PCL-Retaining Total Knee Arthroplasty,” Orthopaedics \& Traumatology, Surgery \& Research, Vol. 95, No. 4, 2009, pp. 254-259. doi:10.1016/j.otsr.2009.03.012

[27] J. S. Gebhard and D. J. Kilgus, "Dislocation of a Posterior Stabilized Total Knee Prosthesis. A Report of Two Cases," Clinical Orthopaedics \& Related Research, Vol. 254, 1990, pp. 225-229.

[28] B. H. Hwang, W. S. Lee, K. K. Park, I. H. Yang and C. D. Han, "Anterior-Posterior Glide Mobile-Bearing Total Knee Arthroplasty Complications Related to Prosthesis Design,” Journal of Arthroplasty, Vol. 26, No. 8, 2011, pp. 1438-1444. doi:10.1016/j.arth.2010.10.007

[29] J. Afriat and F. Larrouy, "Is Dislocation a Specific Risk Inherent in Mobile-Bearing Total Knee Replacements?” Maîtrise Orthopédique MO No88, 1999. http://www.maitrise-orthop.com/viewPage_us.do?id=504

[30] C. H. Huang, J. J. Liau and C. K. Cheng, "Fixed or Mobile-Bearing Total Knee Arthroplasty,” Journal of Orthopaedic Surgery and Research, Vol. 2, No. 1, 2007, pp. 1-8. doi:10.1186/1749-799X-2-1 